\title{
TAS-102 - a new option in the treatment of patients with advanced colorectal cancer after failure or intolerance to other therapies
}

Piotr Skalij", ${ }^{1,}$, Piotr Tokajuk ${ }^{1,2}$, Marek Z. Wojtukiewicz ${ }^{1,2}$

'Department of Oncology, Medical University of Bialystok, Poland

${ }^{2}$ Department of Oncology with Daytime Chemotherapy Unit, Bialystok Oncology Centre, Poland

Correspondence:

Marek Z. Wojtukiewicz

Department of Oncology,

Medical University of Bialystok, Poland

15-027 Bialystok, Ogrodowa 12

Received:

10.03.2020

Accepted:

27.03.2020

DOI: 10.24292/01.OR.120270320

Copyright $\odot$ Medical Education. All rights reserved.

\section{ABSTRACT}

Colorectal cancer is the second cause of death from malignant cancer, following lung cancer. Approximately $35-45 \%$ of patients with colorectal cancer will develop distant metastases over time. Palliative systemic treatment remains the standard for non-operative distant metastases. TAS-102 is a novel orally administered antineoplasmatic thymidine-based nucleoside analog which represents an approved option for the treatment of advanced metastatic colorectal cancer in patients who are refractory, or are not considered canditates for, currently available therapies.

Key words: metastatic colorectal cancer, refractory, fluoropyrimidines, TAS-102 


\section{INTRODUCTION}

Colorectal cancer (CRC) is the second most often diagnosed malignant cancer in Poland. At the same time, it is the second cause of death from malignancies, following lung cancer. Just over a quarter of patients at the time of diagnosis is recognized with the metastatic form of CRC ( $m C R C)$. Additionally, another 35-45\% of patients will develop distant metastases over time [1,2].

Palliative systemic treatment remains the standard for non-operative distant metastases. In the treatment of the first and second lines, a combination of irinotecan or oxaliplatin with fluoropyrimidine is usually used. Moreover, molecularly targeted therapy involving monoclonal antibodies against epidermal growth factor receptor (EGFR) and against vascular endothelial growth factor (VEGF) is used in the treatment of $\mathrm{mCRC}$ [3]. The introduction of the above therapeutic options to clinical practice significantly extended the overall survival (OS) of the patients with mCRC with median OS of up to 30 months [4,5]. However, it should be noted that some patients remain in good general condition after using standard treatment and in case of further deteriorating, additional or effective therapeutic options were not available for these patients. Recent large-scale clinical studies have shown the effectiveness of two new drugs in $\mathrm{mCRC}$ patients after failure or intolerance of mentioned above standard treatment. Both regorafenib and TAS-102 affect the prolongation of OS. The following study discusses the mechanism of action and clinical efficacy of the latter.

\section{THE ANTI-TUMOUR MECHANISM OF ACTION OF TAS-102}

The mechanism of action of TAS-102 is difficult to consider in isolation from other chemotherapeutics that are analogues of pyrimidines. Inhibition of nucleosides is one of the basic mechanisms used in classic cancer therapy for decades. Fluoropyrimidines, such as 5-fluorouracil and its derivatives, are uracil-based nucleic acid analogues that block the enzyme thymidylate synthase (ST). This enzyme catalyses the reaction of methylene-tetrahydrofolate-dependent transformation of deoxyuridine-5'-monophosphate (dUMP), leading to the formation of thymidine-5'-monophosphate (dTMP), which is one of the main substrates for biosynthesis of cellular deoxyribonucleic acid (DNA). The ST-dependent reaction is the only source of dTMP in the cell, therefore it is essential in DNA synthesis [6]. The importance of the ST pathway is emphasized by the fact that ST overexpression is observed in a number of cancers and is associated with worse prognosis [7]. 5-fluorouracil (5-FU) is converted into fluorodeoxyuridine-5'-monophosphate (FdUMP) in the cell, which is then recognized by ST as a false substrate and under- goes a partial reaction catalysed by this enzyme, blocking it by forming a stable enzyme-FdUMP-complex methyl group donor [8]. The accumulation of another 5-FU metabolite, fluorodeoxyuridine triphosphate (FdUTP) together with physiological dUTP in the cell causes their incorrect incorporation into DNA and consequently, during the repair attempt of the DNA, it causes the strand to break resulting in cell death [9]. Fluorouridine triphosphate (FUTP) is the third active anti-cancer metabolite of 5-FU, its incorporation int ribonucleic acid (RNA) leads to its damage [10]. The cytotoxicity of 5-FU associated with DNA damage is more pronounced during long 5-FU infusions, whereas the 5-FU bolus infusion causes the cytotoxicity by RNA damage [11]. Other fluoropyrimidine derivatives, such as capecitabine, tegafur-uracil and S-1 also act through the mechanisms of cell damage mentioned above. They are all converted to $5-\mathrm{FU}$ in vivo $[12,13]$.

TAS-102 is a combination of analogue of thymidine-based nucleoside trifluridine (FTD) and thymidine phosphorylase (FT) inhibitor - typiracil hydrochloride (TC) in a molar ratio of 2:1. The halflife of FTD after intravenous administration is short due to the rapid degradation to its metabolites, i.e. trifluoromethyl deoxyuridine 5-monophosphate (FdTMP) [14]. This metabolite bounds with the active area of the ST becoming its strong inhibitor. Importantly, the inhibition is reversible because, unlike FdUMP, FdTMP does not form a ternary complex with the enzyme, hence the inhibition stops after washing away FTD and metabolites [15-17]. Further phosphorylation of FdTMP results in the formation of metabolite, such as trifluoromethyl deoxyuridine 5-triphosphate (F3dTTP), which can be substituted for DNA $[11,13,18]$. The substitution of F3dTTP to DNA is catalysed by the a subtype DNA polymerase and occurs mainly in the sites opposite to adenine [19]. In the laboratory models, FTD derivatives are incorporated into DNA much more intensively than 5-FU and therefore reach a concentration several times higher than the former [20]. It may be related to the fact, that unlike FdUTP, the F3dTTP is not a substrate for dUTPase - an enzyme that hydrolyzes deoxyuridine triphosphate to deoxyuridine monophosphate, blocking the same incorporation of uracil and its analogues into DNA $[13,19]$. Interestingly, the intensity of FTD incorporation into DNA is much higher in cancer cells when compared to the physiological cells, which is probably associated with increased activity of the pyrimidine metabolic pathway in cancer tissues $[13,19]$. The abilities of DNA to be repaired through the base pair excision, differ significantly in the case of FTD-induced DNA damage in comparison to 5-FU-induced damage. Glycosylases responsible for recognizing and excluding damaged bases that are involved in the removal of uracil and 5-FU, primarily UNG, SMUG1, TDG and MBD4, are inactive in the case when FTD is 
paired with adenine. In the situation of combining FTD with guanine, TDG and MBD4 glycosylases remain active, however, such pairing occurs much less frequently [21].

Also, the second component of TAS-102, TC, has some anti-tumour activity: by inhibiting FT, it reduces angiogenesis. Thy midine phosphorylase exhibits the activity of platelet-derived endothelial cell growth factor and promotes tumour growth through mechanisms associated with endothelial cell migration $[22,23]$. It has been shown in animal models that TC inhibits FT-induced angiogenesis and blocks the development of metastases $[24,25]$. However, it should be noted that using the same doses as during TAS-102 therapy, the described effect of TC on tumour development is probably marginal. The effect of TC on the pharmacokinetics of FTD is much more important. The half-life of FTD after intravenous administration in humans is short and is only 18 minutes [26]. In turn, after oral administration, plasma FTD concentration is very low, which is caused by its degradation by FT, an enzyme that catalyses phosphorolysis, including FTD, in the intestines and liver [27, 28]. The addition of an FT inhibitor, such as TC, to FTD significantly improves its bioavailability and thus clinical effectiveness of TAS-102.

\section{CLINICAL EFFECTIVENESS AND SAFETY OF TAS-102 THERAPY}

The positive effect of TAS-102 on extending the OS of patients with $\mathrm{mCRC}$ was demonstrated in randomized clinical trials: one phase II and two phase III.

112 patients were enrolled in the Phase II study in Japan, the stratification to both arms of the study (TAS-102 vs. placebo) was 2:1. Eligible patients were in a fairly good general condition after not responding to or not tolerating at least two systemic lines of treatment, with the majority of patients being treated with at least three lines of systemic therapy (irinotecan-based chemotherapy, oxaliplatin fluoropyrimidines in all patients, most of them also received anti-EGFR and anti-angiogenic treatment). The inclusion criteria for the study were: the ability of completing the oral therapy, adequate efficiency of bone marrow, liver and kidneys as well as the occurrence of measurable changes. All patients received appropriate symptomatic treatment. The primary endpoint of the study was OS, whose median was 9 months (95\% Cl 7.3-11.3) in the TAS-102 group and 6.6 months (95\% Cl 4.9-8.0) in the placebo group. In the patients treated with TAS-102 haematological complications of at least III grade were observed, half of the group had neutropenia, leukopenia occurred in $28 \%$, and anaemia in $17 \%$ of patients receiving the drug. None of the patients in the control group had grade III or IV neutropenia or leukopenia, while $5 \%$ of the patients had anaemia. In addition, small, but statistically significant differences in the number of adverse events between the arms of the study concerned the occurrence of diarrhoea, nausea and anorexia [29].

Similar results were obtained in the phase III study - RECOURSE (TAS-102 + best supportive care, BSC vs. placebo + BSC). The study included 800 patients with $\mathrm{mCRC}$ previously treated with at least two chemotherapy regimens containing fluoropyrimidine, oxaliplatin, irinotecan and bevacizumab (the above drugs were previously received by all patients included in the study) and anti-EGFR therapy in patients with wild-type KRAS. Nearly $20 \%$ of respondents also received regorafenib as part of previous therapy. Only people in good general condition were qualified for the study, the majority were Caucasian (57\%), slightly more than a half had a KRAS gene mutation. The primary analysis of the results achieved a statistically significant difference at the primary endpoint of OS of 1.8 months (7.1 vs. 5.3 months) in favour of TAS-102 [30]. A recently updated survival analysis increased this difference to 2 months (mOS: 7.2 vs. 5.2 months) [31]. Significant changes were observed regardless of race and mutation status of RAS genes. Also in terms of time to disease progression (PFS) a small but statistically significant difference (2.0 vs. 1.7 months) was achieved, which was also observed in the abovementioned subgroups. In addition, TAS-102 therapy extended the time to deterioration in Eastern Cooperative Oncology Group (ECOG) score compared to BSC, defined as a change from baseline of 0 or 1 to a value of 2 or higher. The difference was 1.7 months (5.7 vs. 4.0 months). Disease control (DCR, complete/partial response or stabilization of the disease assessed at least 6 weeks after randomization) was achieved in $44 \%$ of patients in the TAS-102 group and $16 \%$ of patients in the placebo group $(p<0.001)$. Adverse events $(A E)$ of grade III or higher were more common in the TAS-102 group than in the placebo group ( $69 \%$ vs. $52 \%$ of patients). The differences in the frequency of $\mathrm{AE}$ mainly related to haematological complications: neutropenia ( $38 \%$ vs. $0 \%)$, anaemia ( $18 \%$ vs. $3 \%$ ) and thrombocytopenia ( $5 \%$ vs. $<1 \%$ ). In addition, nausea, vomiting and diarrhoea were slightly more common in patients receiving TAS-102. In turn, no differences were observed in the occurrence of liver or kidney dysfunction, anorexia, stomatitis and cardiac events.

The TERRA study, the second clinical study of phase III, evaluating TAS-102 activity in mCRC patients, was limited to the Asian population only. Similarly to RECOURSE, the study included adults with $\mathrm{mCRC}$ in good general condition (ECOG Score: 0 or 1), who had previously been treated with at least two lines of treatment, regardless of the state of the KRAS gene. Patients were assigned in 
a 2:1 ratio to both arms of the study (TAS-102 + BSC vs. placebo + $\mathrm{BSC})$, the majority of the patients were male, the median age was 58 years (24-81 years), most patients did not have the KRAS gene mutation (63\%) and nearly half of the respondents were treated with biological therapies: anti-VEGF, anti-EGFR or both. The results confirmed the effectiveness of the study drug. The median of OS was significantly longer in the TAS-102 group compared to the placebo group (7.8 vs. 7.1 months, respectively). The analysis of the subgroup showed the benefit of chemotherapy for all parameters, except age. The differences in progression free survival (PFS) (2.0 vs. 1.8 months) and time to treatment failure (TTF) (1.9 vs. 1.8 months) also proved statistical significance. The disease control rate (DCR) was clearly higher in the examined arm ( $44.1 \%$ vs. $14.6 \%)$. The AE of grade III or higher were more common in subjects receiving study medication (45.8\%) compared to the placebo group (10.4\%). The largest differences concerned the incidence of neutropenia (33.2\% vs. $0 \%$ ) and anaemia (17.7 vs. 5.9\%) [32].

Firstly, the differences in endpoint values of both studies III could be explained by an additional stratifying factor in the RECOURSE study, which was the time from diagnosis of metastatic disease. Being an independent factor in OS prolongation, the uneven distribution of this parameter in favour of the placebo group in the TERRA study could have overstated the expected survival. Secondly, the differences could have been due to the disproportions in the availability of prior biological therapy in the populations included in both studies. Unscheduled subgroup analysis in the TERRA study showed an increase in the median OS difference between study arms among patients previously receiving biological therapy. Such patients constituted a significantly smaller percentage of patients compared to patients included in the RECOURSE study.

There is evidence confirming both the safety and the effectiveness of therapy and was provided by retrospective analyses of patients who received TAS-102 in the national expanded access programs. The former was carried out in the Netherlands and a total of 136 patients were analysed. The population of Dutch patients treated with TAS-102 differed from the population studied in the RECOURSE study. Twelve patients (9\%) did not meet the RECOURSE qualification criteria because of ECOG performance grade II, eight patients (6\%) had not previously received at least two systemic treatment lines. In addition, 35 patients had not previously received all standard treatments, primarily regarding the use of bevacizumab (37 patients, 27\%). A total of 43 patients (32\%) were considered as not eligible for treatment according to the RECOURSE study criteria. After a median follow-up of 4.2 months, disease progressed in 120 patients (88\%) and 93 patients died (68\%). The median PFS was 2.1 months (95\% Cl 1.8-2.3), while the median OS was 5.4 months (95\% Cl 4.0-6.9). The analyses of subgroup indicated that the subgroup of patients who received TAS-102 therapy at the early stage of treatment (and for this reason not meeting the RECOURSE criteria, $\mathrm{n}=35$ ) had a higher median OS (8.5 months; $95 \% \mathrm{Cl}, 5.2-11.8$ ) compared to the patients who had previously been treated with all available therapies (mOS 4.7 months; $95 \% \mathrm{Cl} 3.6-5.8$ ). The median OS in patients with ECOG PS 0-1 was 5.9 months $(95 \% \mathrm{Cl}$, 4.4-7.3), compared to 3.2 months (1.4-5.0) in patients with grade II according to ECOG. The safety analysis reported fewer AE of at least $3^{\text {rd }}$ stage compared to data obtained in the RECOURSE study (44\% vs. $69 \%$ ), the most common was neutropenia (44\%). This discrepancy could have been influenced by i.e. higher percentage of patients who received the reduced dosage compared to the registration study ( $23 \%$ vs. $14 \%$ ) [33].

Similar results were provided by the Italian analysis of 341 patients treated in the expanded access program in this country. Patients included in the analysis constituted a similar population as in the RECOURSE study, $2 \%$ of patients did not meet the criteria for ECOG performance status and $6 \%$ did not have the required amount of prior systemic treatment. The median OS achieved was 6.2 months ( $95 \% \mathrm{Cl} 5.4-7.2$ ), while the median PFS was 2.4 months $(95 \% \mathrm{Cl} 2.2-2.6)$. The estimated 1-year OS and the estimated 6-month PFS were $26 \%$ and $19 \%$, respectively. The safety profile coincided with the registration study. Interestingly, the development of at least grade $3^{\text {rd }}$ neutropenia at any time during treatment was associated with both PFS ( $\mathrm{HR}=0.44$; $95 \% \mathrm{Cl} 0.35-0.55 ; \mathrm{p}<0.001)$ and $\mathrm{OS}(\mathrm{HR}=0,35 ; 95 \% \mathrm{Cl} 0.28-0.45$; $p<0.001$ ). After the transformation reducing the impact of the number of cycles on the result, the above relationship was still observed in the case of OS, with a tendency to significance in the PFS range $(p=0.095)$. It is worth noting that more than half of the patients included in the analysis were treated with both TAS-102 and regorafenib. 121 patients received regorafenib before TAS-102, while 61 patients underwent the reverse sequence. There were no differences in the first PFS $(H R=0.96$; $95 \% \mathrm{Cl} 0.71-1.31 ; p=0.808)$, the second PFS (HR = 1.06; $95 \% \mathrm{Cl} 0.75-1.50 ; \mathrm{p}=0.737)$ and $\mathrm{OS}(\mathrm{HR}=0.85 ; 95 \% \mathrm{Cl} 0.85-1.56$; $p=0.388$ ) between two subgroups [34].

\section{PREDICTIVE FACTORS FOR RESPONSE TO TAS-102 TREATMENT}

The above data show that half of the patients treated with TAS-102 do not benefit from this procedure. At the same time, the predictions of response to treatment with this drug are not 
known. A model developed for refractory mCRC patients can help with appropriate selection for therapy. It assesses the probability of death of the patient within the next 12 weeks. It was developed for patients receiving TAS-102 or regorafenib, an oral tyrosine kinase inhibitor also used in this indication. Life expectancy $\geq 12$ weeks was the inclusion criterion for including both drugs in the registration tests and and can also be used in daily clinical practice. The resulting "ColonLife" nomogram includes four variables: ECOG performance status, primary tumour resection, lactate dehydrogenase level and peritoneal involvement (fig. 1) [35]. The value of the nomogram was confirmed in another analysis, including patients treated with the TAS-102 treatment in the extended access program in Italy. In addition, the clinical and molecular characteristics of patients without disease progression after 6 months were compared, the significant variables occurred: ECOG performance status, serum LDH, previous tumour resection, and the time since diagnosis of metastatic disease $(<18$ months vs. $>18$ months). As seen, they mostly coincided with the "ColonLife" model. Interestingly, tumour-related features, such as the number and location of metastases, were widely considered to be a negative prognostic factor in oncology [36].

\section{CONCLUSIONS}

The registration of TAS-102 in the treatment of patients with $\mathrm{mCRC}$ extends the new therapeutic possibilities after failure or intolerance of standard treatment. TAS-102 therapy should be however limited to patients in good and very good ECOG performance status as demonstrated by both registration studies and subsequent clinical analyses. Therapy with TAS-102 was associated with a significantly longer OS and PFS. From the perspective of quality of life, it is also important to observe the increase in time until the deterioration of general performance. Complications of TAS-102 treatment occur in the majority of patients and their profile mainly concerns haematological complications. Studies directly comparing TAS-102 with regorafenib are not available, however, available data confirm the efficacy of the sequential use of both drugs, and the order of such configuration remains irrelevant. The optimal selection of patients for TAS-102 therapy can be supported by the "ColonLife" nomogram, assessing the possibility of 12-week survival of refractory patients with $\mathrm{mCRC}$. There are ongoing studies assessing the effectiveness of pairing TAS-102 with targeted treatment or other cytostatics, including bevacizumab, panitumumab, binimetin-

\section{FIGURE 1.}

A12-week deathnomogram. The nomogram provides amethod to calculate 12-week probability of death after investigator's assessed date of refractory mCRC. Touse, locate primary tumor resection (yes, no), draw a line straight up to the 'Points' axis to determine the score associated to primary tumor resection. Repeat for the other three variables: ECOGPerformance Status (0,1 or 2), LDH value and pres-ence of peritoneal metastases (no, yes). Sum the scores and locate the total score on the 'Total Points' axis. Draw a line straight downwards to the 'Probability' axis to obtain the probability.

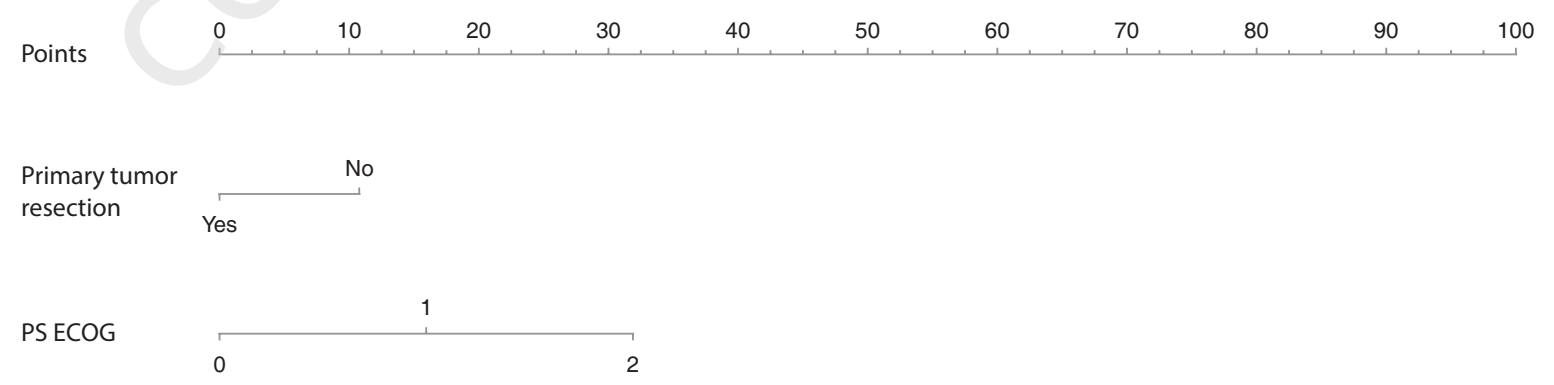

$\mathrm{LDH}(\mathrm{U} / \mathrm{I})$

\begin{tabular}{|c|c|c|c|c|c|c|c|}
\hline 250 & 500 & 1000 & 1500 & 2000 & 2500 & 3000 & 3500 \\
\hline
\end{tabular}

Peritoneal

Metastasis

Yes

No

Total points

T r r

10

20

30

40

50

60

70

80

90

100

$110 \quad 120$

Probability

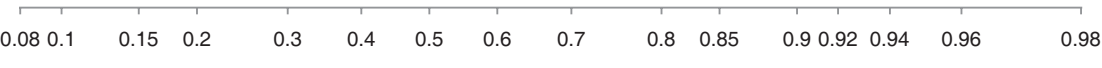


$i b$, also in early lines of treatment. The results obtained so far seem promising and provide solid foundations to continue the research in the future. Currently, TAS-102 monotherapy, thanks to the effectiveness and acceptable toxicity described above, remains an interesting therapeutic option for carefully selected patients with advanced CRC.

\section{References}

1. Wojciechowska U, Czaderny K, Ciuba A et al. Nowotwory złośliwe w Polsce w 2016 roku. Warszawa 2018.

2. Cromheecke M, de Jong KP, Hoekstra HJ. Current treatment for colorectal cancer metastatic to the liver. Eur J Surg Oncol. 1999; 25: 451-63.

3. Hochster HS, Grothey A, Hart L et al. Improved time to treatment failure with an intermittent oxaliplatin strategy: Results of concept. Ann Oncol. 2014; 25: 1172-8.

4. Yoshino T, Arnold D, Taniguchi $\mathrm{H}$ et al. Pan-Asian adapted ESMO consensus guidelines for the management of patients with metastatic colorectal cancer: A JSMO-ESMO initiative endorsed by CSCO, KACO, MOS, SSO and TOS. Ann Oncol. 2018: 29: 44-70.

5. Vogel A, Hofheinz RD, Kubicka $S$ et al. Treatment decisions in metastatic colorectal cancer - Beyond first and second line combination therapies. Cancer Treat Rev. 2017; 59: 54-60.

6. Wilson PM, Danenberg PV, Johnston PG et al. Standing the test of time: targeting thymidylate biosynthesis in cancer therapy. Nat Rev Clin Oncol. 2014; 11: 282-98.

7. Rahman L, Voeller D, Rahman M et al. Thymidylate synthase as an oncogene: a novel role for an essential DNA synthesis enzyme. Cancer Cell. 2004; 5:341-51.

8. Longley DB, Harkin DP, Johnston PG. 5-fluorouracil: mechanisms of action and clinical strategies. Nat Rev Cancer. 2003; 3: 330-8.

9. Wyatt MD, Wilson DM 3rd. Participation of DNA repair in the response to 5-fluorouracil. Cell Mol Life Sci. 2009; 66: 788-99.

10. Glazer RI, Lloyd LS. Association of cell lethality with incorporation of 5-fluorouracil and 5-fluorouridine into nuclear RNA in human colon carcinoma cells in culture. Mol Pharmacol. 1982; 21: 468-73.

11. Aschele C, Sobrero A, Faderan MA et al. Novel mechanism(s) of resistance to 5 -fluorouracil in human colon cancer (HCT-8) sublines following exposure to two different clinically relevant dose schedules. Cancer Res. 1992; 52: 1855-64.

12. Wilson PM, Danenberg PV, Johnston PG et al. Standing the test of time: targeting thymidylate biosynthesis in cancer therapy. Nat Rev Clin Oncol. 2014; 11: 282-98.

13. Lenz HJ, Stintzing S, Loupakis F. TAS-102, a novel antitumor agent: a review of the mechanism of action. Cancer Treat Rev. 2015; 41: 777-83.

14. Dexter DL, Wolberg WH, Ansfield FJ et al. The clinical pharmacology of 5-trifluoromethyl-2'-deoxyuridine. Cancer Res. 1972; 32: 247-53.

15. Temmink $\mathrm{OH}$, Emura T, de Bruin $\mathrm{M}$ et al. Therapeutic potential of the dual-targeted TAS-102 formulation in the treatment of gastrointestinal malignancies. Cancer Sci. 2007; 98: 779-89.

16. Eckstein JW, Foster PG, Finer-Moore J et al. Mechanism-based inhibition of thymidylate synthase by 5-(trifluoromethyl)-2'-deoxyuridine 5'-monophosphate. Biochemistry. 1994; 33: 15086-94.

17. Temmink OH, Comijn EM, Fukushima M et al. Intracellular thymidylate synthase inhibition by trifluorothymidine in FM3A cells. Nucleosides Nucleotides Nucleic Acids. 2004; 23: 1491-4.

18. Heidelberger C, Boohar J, Kampschroer B. Fluorinated pyrimidines. XXIV. In vivo metabolism of 5-trifluoromethyluracil-2-C-14 and 5-trifluoromethyl-2'-deoxyuridine-2-C-14. Cancer Res. 1965; 25: 377-81.

19. Oguchi K, Sakamoto K, Kazuno H et al. TAS-102 treatment results in high trifluridine incorporation into DNA with pyrimidine metabolic pathway markedly up-regulated in cancer. Eur J Cancer. 2014; 50(suppl. 6): Abstract 27.

20. Suzuki N, Emura T, Fukushima M. Mode of action of trifluorothymidine (TFT) against DNA replication and repair enzymes. Int J Oncol. 2011;39: 263-70.

21. Suzuki N, Emura T, Fukushima M. Mode of action of trifluorothymidine (TFT) against DNA replication and repair enzymes. Int J Oncol. 2011;39: 263-70.

22. Moghaddam A, Zhang HT, Fan TP et al. Thymidine phosphorylase is angiogenic and promotes tumour growth. Proc Natl Acad Sci USA. 1995; 92: 998-1002.

23. Hotchkiss KA, Ashton AW, Schwartz EL. Thymidine phosphorylase and 2-deoxyribose stimulate human endothelial cell migration by specific activation of the integrins alpha 5 beta 1 and alpha V beta 3. J Biol Chem. 2003; 278: 19272-9.

24. Takao S, Akiyama SI, Nakajo A et al. Suppression of metastasis by thymidine phosphorylase inhibitor. Cancer Res. 2000; 60: 5345-8.

25. Matsushita S, Nitanda T, Furukawa T et al. The effect of a thymidine phosphorylase inhibitor on angiogenesis and apoptosis in tumors. Cancer Res. 1999; 59: 1911-6.

26. Dexter DL, Wolberg WH, Ansfield FJ et al. The clinical pharmacology of 5-trifluoromethyl-2'-deoxyuridine. Cancer Res. 1972; 32: 247-53.

27. Emura T, Suzuki N, Fujioka A et al. Potentiation of the antitumor activity of alpha, alpha, alpha-trifluorothymidine by the co-administration of an inhibitor of thymidine phosphorylase at a suitable molar ratio in vivo. Int J Oncol. 2005; 27: 449-55.

28. Fukushima M, Suzuki N, Emura T et al. Structure and activity of specific inhibitors of thymidine phosphorylase to potentiate the function of a ntitumor 2'-deoxyribonucleosides. Biochem Pharmacol. 2000; 59: 1227-36.

29. Yoshino T, Mizunuma N, Yamazaki K et al. TAS-102 monotherapy for pretreated metastatic colorectal cancer: a double-blind, randomised, placebo-controlled Phase 2 trial. Lancet Oncol. 2012; 13(10): 993-1001.

30. Mayer RJ, Van Cutsem E, Falcone A et al. Randomized trial of TAS-102 for refractory metastatic colorectal cancer. N Engl J Med. 2015; 372(20): 1909-19.

31. Van Cutsem E, Mayer RJ, Laurent S et al. The subgroups of the Phase III RECOURSE trial of trifluridine/tipiracil (TAS-102) versus placebo with best supportive care in patients with metastatic colorectal cancer. Eur J Cancer. 2018; 90: 63-72.

32. Xu J, Kim TW, Shen L et al. Results of a randomized, double-blind, placebo-controlled, Phase III trial of trifluridine/tipiracil (TAS-102) monotherapy in Asian patients With previously treated metastatic colorectal cancer: the TERRA study. J Clin Oncol. 2018; 36(4): 350-8.

33. Kwakman JJM, Vink G, Vestjens JH et al. Feasibility and effectiveness of trifluridine/tipiracil in metastatic colorectal cancer: real-life data from The Netherlands. Int J Clin Oncol. 2018; 23:482-9.

34. Cremolini C, Rossini D, Martinelli E et al. Trifluridine/tipiracil (TAS-102) in refractory metastatic colorectal cancer: a multicenter register in the frame of the Italian compassionate use program. Oncologist. 2018; 23:1178-87.

35. Pietrantonio F, Miceli R, Rimassa L et al. Estimating 12-week death probability in patients with refractory metastatic colorectal cancer: The Colon Life nomogram. Ann Oncol. 2017; 28: 555-61.

36. Cremolini C, Rossini D, Martinelli E et al. Trifluridine/tipiracil (TAS-102) in refractory metastatic colorectal cancer: a multicenter register in the frame of the Italian compassionate use program. Oncologist. 2018; 23: 1178-87. 